\title{
LEXSEGMENT IDEALS AND THEIR H-POLYNOMIALS
}

\author{
TAKAYUKI HIBI AND KAZUNORI MATSUDA
}

\begin{abstract}
Let $S=K\left[x_{1}, \ldots, x_{n}\right]$ denote the polynomial ring in $n$ variables over a field $K$ with each $\operatorname{deg} x_{i}=1$ and $I \subset S$ a homogeneous ideal of $S$ with $\operatorname{dim} S / I=d$. The Hilbert series of $S / I$ is of the form $h_{S / I}(\lambda) /(1-\lambda)^{d}$, where $h_{S / I}(\lambda)=h_{0}+h_{1} \lambda+h_{2} \lambda^{2}+\cdots+$ $h_{s} \lambda^{s}$ with $h_{s} \neq 0$ is the $h$-polynomial of $S / I$. Given arbitrary integers $r \geq 1$ and $s \geq 1$, a lexsegment ideal $I$ of $S=K\left[x_{1}, \ldots, x_{n}\right]$, where $n \leq \max \{r, s\}+2$, satisfying $\operatorname{reg}(S / I)=r$ and $\operatorname{deg} h_{S / I}(\lambda)=s$ will be constructed.
\end{abstract}

The study on the regularity and the degree of the $h$-polynomial of a monomial ideal done in [3] continues in the present paper and an affirmative answer to [3, Conjecture 0.1] will be given.

Let $S=K\left[x_{1}, \ldots, x_{n}\right]$ denote the polynomial ring in $n$ variables over a field $K$ with each $\operatorname{deg} x_{i}=1$ and $I \subset S$ a homogeneous ideal of $S$ with $\operatorname{dim} S / I=d$. The Hilbert function of $S / I=\bigoplus_{n=0}^{\infty}(S / I)_{n}$ is the numerical function $H(S / I, n)=\operatorname{dim}_{K}(S / I)_{n}$ for $n \in \mathbb{Z}_{\geq 0}$. The Hilbert series of $S / I$ is the formal power series $F(S / I, \lambda)=\sum_{n=0}^{\infty} H(S / I, n) \lambda^{n} \in \mathbb{Z}[[\lambda]]$ of $\{H(S / I, n)\}_{n=0}^{\infty}$. It is known ([2, Theorem 6.1.3]) that $F(R / I, \lambda)$ is of the form

$$
\left(h_{0}+h_{1} \lambda+h_{2} \lambda^{2}+\cdots+h_{s} \lambda^{s}\right) /(1-\lambda)^{d},
$$

where each $h_{i} \in \mathbb{Z}$. We say that

$$
h_{S / I}(\lambda)=h_{0}+h_{1} \lambda+h_{2} \lambda^{2}+\cdots+h_{s} \lambda^{s}
$$

with $h_{s} \neq 0$ is the h-polynomial of $S / I$. Let $\operatorname{reg}(S / I)$ denote the (Castelnuovo-Mumford) regularity [2, p. 48] of $S / I$. In the previous paper [3], given arbitrary integers $r$ and $s$ with $r \geq 1$ and $s \geq 1$, a monomial ideal $I$ of $S=K\left[x_{1}, \ldots, x_{n}\right]$ with $n \gg 0$ for which $\operatorname{reg}(S / I)=r$ and $\operatorname{deg} h_{S / I}(\lambda)=s$ was constructed and it is conjectured that the desired monomial ideal can be chosen to be a strongly stable ideals ([3, Conjecture 0.1]). The purpose of the present paper is to claim that [3, Conjecture 0.1] turns out to be true.

Theorem 0.1. Given arbitrary integers $r \geq 1$ and $s \geq 1$, there exists a lexsegment ideal I of $S=K\left[x_{1}, \ldots, x_{n}\right]$ with $n \leq \max \{r, s\}+2$ for which $\operatorname{reg}(S / I)=r$ and $\operatorname{deg} h_{S / I}(\lambda)=s$.

Proof. (First Step) Let $1 \leq r \leq s$. Let $I$ be the lexsegment ideal [2, p. 103] generated by the monomials

$$
x_{1}^{r+1}, x_{1}^{r} x_{2}, x_{1}^{r} x_{3}, \ldots, x_{1}^{r} x_{s-r+1}
$$

2010 Mathematics Subject Classification. 05E40, $13 \mathrm{H} 10$.

Key words and phrases. Castelnuovo-Mumford regularity, lexsegment ideal, $h$-polynomial. 
of the polynomial ring $S=K\left[x_{1}, \ldots, x_{S-r+1}\right]$. Eliahou-Kervaire formula [2, Corollary 7.2.3] says that $I$ has a linear resolution and $\operatorname{reg}(S / I)=\operatorname{reg}(I)-1=r$. Furthermore, since $I=x_{1}^{r}\left(x_{1}, x_{2}, \ldots, x_{s-r+1}\right)$, one has

$$
\begin{aligned}
F(S / I, \lambda) & =\frac{1}{(1-\lambda)^{s-r+1}}-\left(\frac{\lambda^{r}}{(1-\lambda)^{s-r+1}}-\lambda^{r}\right) \\
& =\frac{1+\lambda+\cdots+\lambda^{r-1}+\lambda^{r}(1-\lambda)^{s-r}}{(1-\lambda)^{s-r}} .
\end{aligned}
$$

Hence $\operatorname{deg} h_{S / I}(\lambda)=s$, as desired.

(Second Step) Let $1 \leq s<r$. We introduce the sequence $\left\{a_{n}\right\}_{n=0}^{\infty}$ which is

$$
a_{0}=1, a_{1}=\cdots=a_{s-1}=r+2, a_{s}=\cdots=a_{r}=\cdots=r+1 .
$$

Since the sequence $\left\{a_{n}\right\}_{n=0}^{\infty}$ satisfy the Macaulay condition [2, Theorem 6.3.8], it follows that there exists a homogeneous ideal $I \subset S=K\left[x_{1}, \ldots, x_{r+2}\right]$ for which $H(S / I, n)=a_{n}$ for $n \in \mathbb{Z}_{\geq 0}$. Let $I^{\text {lex }} \subset S$ denote the lexsegment ideal for which the Hilbert function of $I^{\text {lex }}$ coincides with that of $I$ ([2, Theorem 6.3.1]). Now, if $j \geq r+1$, then $j-r+1 \geq 1$ and the Macaulay expansion ([2, Lemma 6.3.4]) of $h_{j}$ is

$$
h_{j}=r+1=\left(\begin{array}{l}
j \\
j
\end{array}\right)+\left(\begin{array}{l}
j-1 \\
j-1
\end{array}\right)+\cdots+\left(\begin{array}{l}
j-r+1 \\
j-r+1
\end{array}\right) .
$$

Hence

$$
h_{j}^{\langle j\rangle}=\left(\begin{array}{l}
j+1 \\
j+1
\end{array}\right)+\left(\begin{array}{l}
j \\
j
\end{array}\right)+\cdots+\left(\begin{array}{l}
j-r+2 \\
j-r+2
\end{array}\right)=r+1=h_{j+1} .
$$

Furthermore, the Macaulay expansion of $h_{r}$ is

$$
h_{r}=r+1=\left(\begin{array}{c}
r+1 \\
r
\end{array}\right)
$$

Hence

$$
h_{r}^{\langle r\rangle}=\left(\begin{array}{l}
r+2 \\
r+1
\end{array}\right)=r+2>h_{r+1} .
$$

It then follows from the proof of [2, Theorem 6.3.1] that the maximal degree of the monomials belonging to the minimal system of generators of $I^{\text {lex }}$ is $r+1$. Again, EliahouKervaire formula [2, Corollary 7.2.3] says that $\operatorname{reg}(R / I)=\operatorname{reg}(I)-1=(r+1)-1=r$. On the other hand,

$$
\begin{aligned}
F(S / I, \lambda) & =1+(r+2)\left(\lambda+\lambda^{2}+\cdots+\lambda^{s-1}\right)+\frac{(r+1) \lambda^{s}}{1-\lambda} \\
& =1+\frac{(r+2) \lambda\left(1-\lambda^{s-1}\right)}{1-\lambda}+\frac{(r+1) \lambda^{s}}{1-\lambda} \\
& =\frac{1+(r+1) \lambda-\lambda^{s}}{1-\lambda}
\end{aligned}
$$

Hence $\operatorname{deg} h_{S / I}(\lambda)=s$, as required. 
Example 0.2. Let $s=2$ and $r=4$. Then the lexsegment ideal $I^{\text {lex }} \subset S=K\left[x_{1}, \ldots, x_{6}\right]$ with the Hilbert function $1,6,5,5,5, \ldots$ is the monomial ideal generated by

$$
\begin{gathered}
x_{1}^{2}, x_{1} x_{2}, x_{1} x_{3}, x_{1} x_{4}, x_{1} x_{5}, x_{1} x_{6}, x_{2}^{2}, x_{2} x_{3}, x_{2} x_{4}, x_{2} x_{5}, x_{2} x_{6}, \\
x_{3}^{2}, x_{3} x_{4}, x_{3} x_{5}, x_{3} x_{6}, x_{4}^{2}, x_{4} x_{5}^{2}, x_{4} x_{5} x_{6}, x_{4} x_{6}^{3}, x_{5}^{5} .
\end{gathered}
$$

One has $\operatorname{dim} S / I^{\text {lex }}=1$ and $\operatorname{depth} S / I^{\text {lex }}=0$. Its Betti table is

$$
\begin{array}{rrrrrrr}
1 & . & . & . & . & . & . \\
. & 16 & 47 & 63 & 46 & 18 & 3 \\
. & 2 & 9 & 16 & 14 & 6 & 1 \\
. & 1 & 5 & 10 & 10 & 5 & 1 \\
. & 1 & 4 & 6 & 4 & 1 & .
\end{array}
$$

Question 0.3. Find all possible sequences $(d, e, r, s) \in \mathbb{Z}_{\geq 0}$ with $d \geq e \geq 0, r \geq 1, s \geq 1$ and $s-r \leq d-e$ for which there exists a homogeneous ideal $I \subset S=K\left[x_{1}, \ldots, x_{n}\right]$ with $n \gg 0$ satisfying

$$
\operatorname{dim} S / I=d, \operatorname{depth} S / I=e, \operatorname{reg}(S / I)=r, \operatorname{deg} h_{S / I}(\lambda)=s .
$$

On the other hand, in general, it is known ([4, Corollary B.4.1]) that one has

$$
\operatorname{deg} h_{S / I}(\lambda)-\operatorname{reg}(S / I) \leq \operatorname{dim} S / I-\operatorname{depth} S / I
$$

for each homogeneous ideal $I$ of $S=K\left[x_{1}, \ldots, x_{n}\right]$.

Remark 0.4. A few remarks are collected.

(1) Let $1 \leq s<r$. Let $I^{\text {lex }}$ be the lexsegment ideal of $S=K\left[x_{1}, \ldots, x_{r+2}\right]$ which appears in Second Step of the proof of Theorem 0.1 . Then $\operatorname{dim} S / I^{\text {lex }}=1, \operatorname{depth} S / I^{\text {lex }}=0$, $\operatorname{reg}\left(S / I^{\text {lex }}\right)=r$ and $\operatorname{deg} h_{S / I^{\text {lex }}}(\lambda)=s$. As a result, Question 0.3 can be solved, when $d=1, e=0$ and $1 \leq s<r$.

(2) Let $1 \leq s<r$. Let $I=I_{r-s}+\left(u_{1} u_{2} \cdots u_{s}\right)$ be the monomial ideal of $S$ which appears in the proof of [3, Theorem 1.2], where

$$
S=K\left[x, y_{1}, \ldots, y_{r-s}, z_{1}, \ldots, z_{r-s+1}, u_{1}, u_{2}, \ldots, u_{s}\right] .
$$

Then $\operatorname{dim} S / I=r, \operatorname{depth} S / I=r-1, \operatorname{reg}(S / I)=r$ and $\operatorname{deg} h_{S / I}(\lambda)=s$. As a result, Question 0.3 can be also solved, when $d=r, e=r-1$ and $1 \leq s<r$.

(3) Let $I$ be the monomial ideals of $S=K\left[x_{1}, x_{2}, x_{3}, x_{4}, x_{5}\right]$ generated by

$$
\begin{gathered}
x_{1}^{2}, x_{1} x_{2}, x_{1} x_{3}, x_{1} x_{4}, x_{1} x_{5}, x_{2}^{2}, x_{2} x_{3}^{2}, x_{2} x_{3} x_{4}, x_{2} x_{3} x_{5}, \\
x_{2} x_{4}^{3}, x_{2} x_{4}^{2} x_{5}, x_{2} x_{4} x_{5}^{3}, x_{2} x_{5}^{4}, x_{3}^{6}, x_{3}^{5} x_{4}, x_{3}^{5} x_{5}, x_{3}^{4} x_{4}^{3} .
\end{gathered}
$$

Then $I$ is a lexsegment ideal and $\operatorname{dim} S / I=2, \operatorname{depth} S / I=0, \operatorname{reg}(S / I)=6$ and $\operatorname{deg} h_{S / I}(\lambda)=1$. It would be of interest to find a lexsegment ideal $I$ of $S=$ $K\left[x_{1}, \ldots, x_{n}\right]$ with $n \gg 0$ satisfying $\operatorname{dim} S / I-\operatorname{depth} S / I=c$ and $\operatorname{deg} h_{S / I}(\lambda)<$ $\operatorname{reg}(S / I)$ for all $c \geq 0$.

(4) Let $I$ be a monomial ideal of $S=K[x, y]$. By virtue of [1, Theorem 2.4], one can easily see that 
- $S / I$ is Cohen-Macaulay if and only if $\operatorname{deg} h_{S / I}(\lambda)=\operatorname{reg}(S / I)$;

- $S / I$ is not Cohen-Macaulay if and only if $\operatorname{deg} h_{S / I}(\lambda)=\operatorname{reg}(S / I)+1$.

Thus in particular one has $\operatorname{deg} h_{S / I}(\lambda) \geq \operatorname{reg}(S / I)$ for all monomial ideals $I$ of $S=K[x, y]$.

(5) It might be a reasonable question to find a natural class of monomial ideals $I$ of $S=K\left[x_{1}, \ldots, x_{n}\right]$ for which (i) $S / I$ is not Cohen-Macaulay, (ii) $I$ does not have a pure resolution and (iii) $\operatorname{deg} h_{S / I}(\lambda)-\operatorname{reg}(S / I)=\operatorname{dim} S / I-\operatorname{depth} S / I$.

Acknowledgements During the participation of the first author in the workshop New Trends in Syzygies, organized by Jason McCullough (Iowa State University) and Giulio Caviglia (Purdue University), Banff International Research Station for Mathematical Innovation and Discovery, Banff, Canada, June 24 - 29, 2018, a motive for writing the present paper arose from an informal conversation with Marc Chardin. Special thanks are due to the BIRS for providing the participants with a wonderful atmosphere for mathematics. The first author is partially supported by JSPS KAKENHI 26220701. The second author is partially supported by JSPS KAKENHI 17K14165.

\section{REFERENCES}

[1] A. Conca and J. Herzog, Castelnuovo-Mumford regularity of products of ideals, Collect. Math. 54 (2003), 137-152.

[2] J. Herzog and T. Hibi, Monomial ideals, Graduate Texts in Mathematics 260, Springer, London, 2010.

[3] T. Hibi and K. Matsuda, Regularity and $h$-polynomials of monomial ideals, Math. Nachr., in press.

[4] W. V. Vasconcelos, Computational Methods in Commutative Algebra and Algebraic Geometry, Springer-Verlag, 1998.

Takayuki Hibi, Department of Pure and Applied Mathematics, Graduate School of INFORMATION SCIENCE AND TECHNOLOGY, OSAKA UNIVERSiTy, SUITA, OSAKA 565-0871, JAPAN

E-mail address: hibi@math.sci.osaka-u.ac.jp

Kazunori Matsuda, Kitami Institute of Technology, Kitami, HokKaido 090-8507, JAPAN E-mail address: kaz-matsuda@mail.kitami-it.ac.jp 